\title{
SPECIAL PAIRS OF PYTHAGOREAN TRIANGLES AND DHURUVA NUMBERS OF ORDERS 4, 5 AND 6
}

\author{
M.A.Gopalan ${ }^{1}$, S. Vidhyalakshmi ${ }^{2}$, N.Thiruniraiselvi ${ }^{3}$, R.Presenna ${ }^{4}$ \\ ${ }^{1}$ Professor, Department of Mathematics, SIGC, Trichy-620002, Tamilnadu, India \\ ${ }^{2}$ Professor, Department of Mathematics, SIGC, Trichy-620002, Tamilnadu, India \\ ${ }^{3}$ Research scholar, Department of Mathematics, SIGC, Trichy-620002, Tamilnadu, India \\ ${ }^{4}$ M.Phil student, Department of Mathematics, SIGC, Trichy-620002, Tamilnadu, India
}

\begin{abstract}
This paper concerns with the problem of obtaining pairs of pythagorean triangles, where, in each pair, the difference between their perimeters is represented by 6174(Dhuruva number of order 4),61974,82962,63954(Dhuruva number of order 5), 631764(Dhuruva number of order 6). Also, we present the number of pairs of primitive and non-primitive Pythagorean triangles.
\end{abstract}

Key words: Pairs of Pythagorean triangles, Dhuruva number, primitive and non-primitive Pythagorean triangles.

2010 Mathematics Subject Classification: 11D09, 11Y50, 11-04.

$* * *$

\section{INTRODUCTION}

The fascinating branch of mathematics is the theory of numbers where in Pythagorean triangles have been a matter of interest to various mathematicians and to the lovers of mathematics, because it is a treasure house in which the search for many hidden connection is a treasure hunt. For a rich variety of fascinating problems one may refer [1-17].A careful observer of patterns may note that there is a one to one correspondence between the polygonal numbers and the number of sides of the polygon. Apart from the above patterns we have some more fascinating patterns of numbers namely Jarasandha numbers, Nasty numbers and Dhuruva numbers. These numbers have been presented in [18-21].

In [22-24], special Pythagorean triangles connected with polygonal numbers and Nasty numbers are obtained. Recently in [25], special Pythagorean triangles in connection with Hardy Ramanujan number 1729 are exhibited. In [26], Pythagorean triangles in connections with 5-digit Dhuruva numbers are presented.

In this communication, we search for pairs of Pythagorean triangles, such that in each pair, the difference between their perimeters is the Dhuruva number.

\section{Basic Definitons}

\section{Definition 1:}

The ternary quadratic Diophantine equation given by $\mathrm{x}^{2}+\mathrm{y}^{2}=\mathrm{z}^{2}$ is known as Pythagorean equation where $\mathrm{x}$, $\mathrm{y}, \mathrm{z}$ are natural numbers. The above equation is also referred to as Pythagorean triangle and denote it by $\mathrm{T}(\mathrm{x}, \mathrm{y}, \mathrm{z})$.
Also, in Pythagorean triangle $\mathrm{T}(\mathrm{x}, \mathrm{y}, \mathrm{z}): \mathrm{x}^{2}+\mathrm{y}^{2}=\mathrm{z}^{2}, \mathrm{x}$ and $\mathrm{y}$ are called its legs and $\mathrm{z}$ its hypotenuse.

\section{Definition 2:}

Most cited solution of the Pythagorean equation is $\mathrm{x}=\mathrm{m}^{2}-\mathrm{n}^{2}, \mathrm{y}=2 \mathrm{mn}, \mathrm{z}=\mathrm{m}^{2}+\mathrm{n}^{2}, \quad$ where $m>n>0$. This solution is called primitive, if $\mathrm{m}, \mathrm{n}$ are of opposite parity and $\operatorname{gcd}(\mathrm{m}, \mathrm{n})=1$.

\section{Definition 3: Dhuruva Numbers}

The numbers which do not change when we perform a single operation or a sequence of operations are known as Dhuruva numbers.

\section{METHOD OF ANALYSIS}

Let $\mathrm{PT}_{1}, \mathrm{PT}_{2}$ be two distinct Pythagorean triangles with generators $\mathrm{m}, \mathrm{q}(\mathrm{m}>\mathrm{q}>0)$, and $\mathrm{p}, \mathrm{q}(\mathrm{p}>\mathrm{q}>0)$ respectively.Let $\mathrm{P}_{1}, \mathrm{P}_{2}$ be the perimeters of $\mathrm{PT}_{1}, \mathrm{PT}_{2}$ such that $\quad \mathrm{P}_{1}-\mathrm{P}_{2}=4$-digit Dhuruva numer 6174

The above relation leads to the equation

$$
(2 m+q)^{2}-(2 p+q)^{2}=12348
$$

After performing numerical computations, it is noted that there are 514 distinct values for $\mathrm{m}, \mathrm{p}$ and $\mathrm{q}$ satisfying (1). For simplicity and clear understanding, we have presented below in table 1 the values of $\mathrm{m}, \mathrm{p}, \mathrm{q}, \mathrm{P}_{1}$ and $\mathrm{P}_{2}$.

\begin{tabular}{|l|l|l|l|l|}
\hline $\mathrm{m}$ & $\mathrm{q}$ & $\mathrm{p}$ & $\mathrm{P} 1$ & $\mathrm{P} 2$ \\
\hline 1030 & 1028 & 1029 & 4239480 & 4233306 \\
\hline 1031 & 1026 & 1030 & 4241534 & 4235360 \\
\hline
\end{tabular}




\begin{tabular}{|c|c|c|c|c|c|c|c|c|c|}
\hline 1032 & 1024 & 1031 & 4243584 & 4237410 & 1090 & 908 & 1089 & 4355640 & 4349466 \\
\hline 1033 & 1022 & 1032 & 4245630 & 4239456 & 1091 & 906 & 1090 & 4357454 & 4351280 \\
\hline 1034 & 1020 & 1033 & 4247672 & 4241498 & 1092 & 904 & 1091 & 4359264 & 4353090 \\
\hline 1035 & 1018 & 1034 & 4249710 & 4243536 & 1093 & 902 & 1092 & 4361070 & 4354896 \\
\hline 1036 & 1016 & 1035 & 4251744 & 4245570 & 1094 & 900 & 1093 & 4362872 & 4356698 \\
\hline 1037 & 1014 & 1036 & 4253774 & 4247600 & 1095 & 898 & 1094 & 4364670 & 4358496 \\
\hline 1038 & 1012 & 1037 & 4255800 & 4249626 & 1096 & 896 & 1095 & 4366464 & 4360290 \\
\hline 1039 & 1010 & 1038 & 4257822 & 4251648 & 1097 & 894 & 1096 & 4368254 & 4362080 \\
\hline 1040 & 1008 & 1039 & 4259840 & 4253666 & 1098 & 892 & 1097 & 4370040 & 4363866 \\
\hline 1041 & 1006 & 1040 & 4261854 & 4255680 & 1099 & 890 & 1098 & 4371822 & 4365648 \\
\hline 1042 & 1004 & 1041 & 4263864 & 4257690 & 1100 & 888 & 1099 & 4373600 & 4367426 \\
\hline 1043 & 1002 & 1042 & 4265870 & 4259696 & 1101 & 886 & 1100 & 4375374 & 4369200 \\
\hline 1044 & 1000 & 1043 & 4267872 & 4261698 & 1102 & 884 & 1101 & 4377144 & 4370970 \\
\hline 1045 & 998 & 1044 & 4269870 & 4263696 & 1103 & 882 & 1102 & 4378910 & 4372736 \\
\hline 1046 & 996 & 1045 & 4271864 & 4265690 & 1104 & 880 & 1103 & 4380672 & 4374498 \\
\hline 1047 & 994 & 1046 & 4273854 & 4267680 & 1105 & 878 & 1104 & 4382430 & 4376256 \\
\hline 1048 & 992 & 1047 & 4275840 & 4269666 & 1106 & 876 & 1105 & 4384184 & 4378010 \\
\hline 1049 & 990 & 1048 & 4277822 & 4271648 & 1107 & 874 & 1106 & 4385934 & 4379760 \\
\hline 1050 & 988 & 1049 & 4279800 & 4273626 & 1108 & 872 & 1107 & 4387680 & 4381506 \\
\hline 1051 & 986 & 1050 & 4281774 & 4275600 & 1109 & 870 & 1108 & 4389422 & 4383248 \\
\hline 1052 & 984 & 1051 & 4283744 & 4277570 & 1110 & 868 & 1109 & 4391160 & 4384986 \\
\hline 1053 & 982 & 1052 & 4285710 & 4279536 & 1111 & 866 & 1110 & 4392894 & 4386720 \\
\hline 1054 & 980 & 1053 & 4287672 & 4281498 & 1112 & 864 & 1111 & 4394624 & 4388450 \\
\hline 1055 & 978 & 1054 & 4289630 & 4283456 & 1113 & 862 & 1112 & 4396350 & 4390176 \\
\hline 1056 & 976 & 1055 & 4291584 & 4285410 & 1114 & 860 & 1113 & 4398072 & 4391898 \\
\hline 1057 & 974 & 1056 & 4293534 & 4287360 & 1115 & 858 & 1114 & 4399790 & 4393616 \\
\hline 1058 & 972 & 1057 & 4295480 & 4289306 & 1116 & 856 & 1115 & 4401504 & 4395330 \\
\hline 1059 & 970 & 1058 & 4297422 & 4291248 & 1117 & 854 & 1116 & 4403214 & 4397040 \\
\hline 1060 & 968 & 1059 & 4299360 & 4293186 & 1118 & 852 & 1117 & 4404920 & 4398746 \\
\hline 1061 & 966 & 1060 & 4301294 & 4295120 & 1119 & 850 & 1118 & 4406622 & 4400448 \\
\hline 1062 & 964 & 1061 & 4303224 & 4297050 & 1120 & 848 & 1119 & 4408320 & 4402146 \\
\hline 1063 & 962 & 1062 & 4305150 & 4298976 & 1121 & 846 & 1120 & 4410014 & 4403840 \\
\hline 1064 & 960 & 1063 & 4307072 & 4300898 & 1122 & 844 & 1121 & 4411704 & 4405530 \\
\hline 1065 & 958 & 1064 & 4308990 & 4302816 & 1123 & 842 & 1122 & 4413390 & 4407216 \\
\hline 1066 & 956 & 1065 & 4310904 & 4304730 & 1124 & 840 & 1123 & 4415072 & 4408898 \\
\hline 1067 & 954 & 1066 & 4312814 & 4306640 & 1125 & 838 & 1124 & 4416750 & 4410576 \\
\hline 1068 & 952 & 1067 & 4314720 & 4308546 & 1126 & 836 & 1125 & 4418424 & 4412250 \\
\hline 1069 & 950 & 1068 & 4316622 & 4310448 & 1127 & 834 & 1126 & 4420094 & 4413920 \\
\hline 1070 & 948 & 1069 & 4318520 & 4312346 & 1128 & 832 & 1127 & 4421760 & 4415586 \\
\hline 1071 & 946 & 1070 & 4320414 & 4314240 & 1129 & 830 & 1128 & 4423422 & 4417248 \\
\hline 1072 & 944 & 1071 & 4322304 & 4316130 & 1130 & 828 & 1129 & 4425080 & 4418906 \\
\hline 1073 & 942 & 1072 & 4324190 & 4318016 & 1131 & 826 & 1130 & 4426734 & 4420560 \\
\hline 1074 & 940 & 1073 & 4326072 & 4319898 & 1132 & 824 & 1131 & 4428384 & 4422210 \\
\hline 1075 & 938 & 1074 & 4327950 & 4321776 & 1133 & 822 & 1132 & 4430030 & 4423856 \\
\hline 1076 & 936 & 1075 & 4329824 & 4323650 & 1134 & 820 & 1133 & 4431672 & 4425498 \\
\hline 1077 & 934 & 1076 & 4331694 & 4325520 & 1135 & 818 & 1134 & 4433310 & 4427136 \\
\hline 1078 & 932 & 1077 & 4333560 & 4327386 & 1136 & 816 & 1135 & 4434944 & 4428770 \\
\hline 1079 & 930 & 1078 & 4335422 & 4329248 & 1137 & 814 & 1136 & 4436574 & 4430400 \\
\hline 1080 & 928 & 1079 & 4337280 & 4331106 & 1138 & 812 & 1137 & 4438200 & 4432026 \\
\hline 1081 & 926 & 1080 & 4339134 & 4332960 & 1139 & 810 & 1138 & 4439822 & 4433648 \\
\hline 1082 & 924 & 1081 & 4340984 & 4334810 & 1140 & 808 & 1139 & 4441440 & 4435266 \\
\hline 1083 & 922 & 1082 & 4342830 & 4336656 & 1141 & 806 & 1140 & 4443054 & 4436880 \\
\hline 1084 & 920 & 1083 & 4344672 & 4338498 & 1142 & 804 & 1141 & 4444664 & 4438490 \\
\hline 1085 & 918 & 1084 & 4346510 & 4340336 & 1143 & 802 & 1142 & 4446270 & 4440096 \\
\hline 1086 & 916 & 1085 & 4348344 & 4342170 & 1144 & 800 & 1143 & 4447872 & 4441698 \\
\hline 1087 & 914 & 1086 & 4350174 & 4344000 & 1145 & 798 & 1144 & 4449470 & 4443296 \\
\hline 1088 & 912 & 1087 & 4352000 & 4345826 & 1146 & 796 & 1145 & 4451064 & 4444890 \\
\hline 1089 & 910 & 1088 & 4353822 & 4347648 & 1147 & 794 & 1146 & 4452654 & 4446480 \\
\hline
\end{tabular}




\begin{tabular}{|c|c|c|c|c|c|c|c|c|c|}
\hline 1148 & 792 & 1147 & 4454240 & 4448066 & 1206 & 676 & 1205 & 4539384 & 4533210 \\
\hline 1149 & 790 & 1148 & 4455822 & 4449648 & 1207 & 674 & 1206 & 4540734 & 4534560 \\
\hline 1150 & 788 & 1149 & 4457400 & 4451226 & 1208 & 672 & 1207 & 4542080 & 4535906 \\
\hline 1151 & 786 & 1150 & 4458974 & 4452800 & 1209 & 670 & 1208 & 4543422 & 4537248 \\
\hline 1152 & 784 & 1151 & 4460544 & 4454370 & 1210 & 668 & 1209 & 4544760 & 4538586 \\
\hline 1153 & 782 & 1152 & 4462110 & 4455936 & 1211 & 666 & 1210 & 4546094 & 4539920 \\
\hline 1154 & 780 & 1153 & 4463672 & 4457498 & 1212 & 664 & 1211 & 4547424 & 4541250 \\
\hline 1155 & 778 & 1154 & 4465230 & 4459056 & 1213 & 662 & 1212 & 4548750 & 4542576 \\
\hline 1156 & 776 & 1155 & 4466784 & 4460610 & 1214 & 660 & 1213 & 4550072 & 4543898 \\
\hline 1157 & 774 & 1156 & 4468334 & 4462160 & 1215 & 658 & 1214 & 4551390 & 4545216 \\
\hline 1158 & 772 & 1157 & 4469880 & 4463706 & 1216 & 656 & 1215 & 4552704 & 4546530 \\
\hline 1159 & 770 & 1158 & 4471422 & 4465248 & 1217 & 654 & 1216 & 4554014 & 4547840 \\
\hline 1160 & 768 & 1159 & 4472960 & 4466786 & 1218 & 652 & 1217 & 4555320 & 4549146 \\
\hline 1161 & 766 & 1160 & 4474494 & 4468320 & 1219 & 650 & 1218 & 4556622 & 4550448 \\
\hline 1162 & 764 & 1161 & 4476024 & 4469850 & 1220 & 648 & 1219 & 4557920 & 4551746 \\
\hline 1163 & 762 & 1162 & 4477550 & 4471376 & 1221 & 646 & 1220 & 4559214 & 4553040 \\
\hline 1164 & 760 & 1163 & 4479072 & 4472898 & 1222 & 644 & 1221 & 4560504 & 4554330 \\
\hline 1165 & 758 & 1164 & 4480590 & 4474416 & 1223 & 642 & 1222 & 4561790 & 4555616 \\
\hline 1166 & 756 & 1165 & 4482104 & 4475930 & 1224 & 640 & 1223 & 4563072 & 4556898 \\
\hline 1167 & 754 & 1166 & 4483614 & 4477440 & 1225 & 638 & 1224 & 4564350 & 4558176 \\
\hline 1168 & 752 & 1167 & 4485120 & 4478946 & 1226 & 636 & 1225 & 4565624 & 4559450 \\
\hline 1169 & 750 & 1168 & 4486622 & 4480448 & 1227 & 634 & 1226 & 4566894 & 4560720 \\
\hline 1170 & 748 & 1169 & 4488120 & 4481946 & 1228 & 632 & 1227 & 4568160 & 4561986 \\
\hline 1171 & 746 & 1170 & 4489614 & 4483440 & 1229 & 630 & 1228 & 4569422 & 4563248 \\
\hline 1172 & 744 & 1171 & 4491104 & 4484930 & 1230 & 628 & 1229 & 4570680 & 4564506 \\
\hline 1173 & 742 & 1172 & 4492590 & 4486416 & 1231 & 626 & 1230 & 4571934 & 4565760 \\
\hline 1174 & 740 & 1173 & 4494072 & 4487898 & 1232 & 624 & 1231 & 4573184 & 4567010 \\
\hline 1175 & 738 & 1174 & 4495550 & 4489376 & 1233 & 622 & 1232 & 4574430 & 4568256 \\
\hline 1176 & 736 & 1175 & 4497024 & 4490850 & 1234 & 620 & 1233 & 4575672 & 4569498 \\
\hline 1177 & 734 & 1176 & 4498494 & 4492320 & 1235 & 618 & 1234 & 4576910 & 4570736 \\
\hline 1178 & 732 & 1177 & 4499960 & 4493786 & 1236 & 616 & 1235 & 4578144 & 4571970 \\
\hline 1179 & 730 & 1178 & 4501422 & 4495248 & 1237 & 614 & 1236 & 4579374 & 4573200 \\
\hline 1180 & 728 & 1179 & 4502880 & 4496706 & 1238 & 612 & 1237 & 4580600 & 4574426 \\
\hline 1181 & 726 & 1180 & 4504334 & 4498160 & 1239 & 610 & 1238 & 4581822 & 4575648 \\
\hline 1182 & 724 & 1181 & 4505784 & 4499610 & 1240 & 608 & 1239 & 4583040 & 4576866 \\
\hline 1183 & 722 & 1182 & 4507230 & 4501056 & 1241 & 606 & 1240 & 4584254 & 4578080 \\
\hline 1184 & 720 & 1183 & 4508672 & 4502498 & 1242 & 604 & 1241 & 4585464 & 4579290 \\
\hline 1185 & 718 & 1184 & 4510110 & 4503936 & 1243 & 602 & 1242 & 4586670 & 4580496 \\
\hline 1186 & 716 & 1185 & 4511544 & 4505370 & 1244 & 600 & 1243 & 4587872 & 4581698 \\
\hline 1187 & 714 & 1186 & 4512974 & 4506800 & 1245 & 598 & 1244 & 4589070 & 4582896 \\
\hline 1188 & 712 & 1187 & 4514400 & 4508226 & 1246 & 596 & 1245 & 4590264 & 4584090 \\
\hline 1189 & 710 & 1188 & 4515822 & 4509648 & 1247 & 594 & 1246 & 4591454 & 4585280 \\
\hline 1190 & 708 & 1189 & 4517240 & 4511066 & 1248 & 592 & 1247 & 4592640 & 4586466 \\
\hline 1191 & 706 & 1190 & 4518654 & 4512480 & 1249 & 590 & 1248 & 4593822 & 4587648 \\
\hline 1192 & 704 & 1191 & 4520064 & 4513890 & 1250 & 588 & 1249 & 4595000 & 4588826 \\
\hline 1193 & 702 & 1192 & 4521470 & 4515296 & 1251 & 586 & 1250 & 4596174 & 4590000 \\
\hline 1194 & 700 & 1193 & 4522872 & 4516698 & 1252 & 584 & 1251 & 4597344 & 4591170 \\
\hline 1195 & 698 & 1194 & 4524270 & 4518096 & 1253 & 582 & 1252 & 4598510 & 4592336 \\
\hline 1196 & 696 & 1195 & 4525664 & 4519490 & 1254 & 580 & 1253 & 4599672 & 4593498 \\
\hline 1197 & 694 & 1196 & 4527054 & 4520880 & 1255 & 578 & 1254 & 4600830 & 4594656 \\
\hline 1198 & 692 & 1197 & 4528440 & 4522266 & 1256 & 576 & 1255 & 4601984 & 4595810 \\
\hline 1199 & 690 & 1198 & 4529822 & 4523648 & 1257 & 574 & 1256 & 4603134 & 4596960 \\
\hline 1200 & 688 & 1199 & 4531200 & 4525026 & 1258 & 572 & 1257 & 4604280 & 4598106 \\
\hline 1201 & 686 & 1200 & 4532574 & 4526400 & 1259 & 570 & 1258 & 4605422 & 4599248 \\
\hline 1202 & 684 & 1201 & 4533944 & 4527770 & 1260 & 568 & 1259 & 4606560 & 4600386 \\
\hline 1203 & 682 & 1202 & 4535310 & 4529136 & 1261 & 566 & 1260 & 4607694 & 4601520 \\
\hline 1204 & 680 & 1203 & 4536672 & 4530498 & 1262 & 564 & 1261 & 4608824 & 4602650 \\
\hline 1205 & 678 & 1204 & 4538030 & 4531856 & 1263 & 562 & 1262 & 4609950 & 4603776 \\
\hline
\end{tabular}




\begin{tabular}{|l|l|l|l|l|}
\hline 1264 & 560 & 1263 & 4611072 & 4604898 \\
\hline 1265 & 558 & 1264 & 4612190 & 4606016 \\
\hline 1266 & 556 & 1265 & 4613304 & 4607130 \\
\hline 1267 & 554 & 1266 & 4614414 & 4608240 \\
\hline 1268 & 552 & 1267 & 4615520 & 4609346 \\
\hline 1269 & 550 & 1268 & 4616622 & 4610448 \\
\hline 1270 & 548 & 1269 & 4617720 & 4611546 \\
\hline 1271 & 546 & 1270 & 4618814 & 4612640 \\
\hline 1272 & 544 & 1271 & 4619904 & 4613730 \\
\hline 1273 & 542 & 1272 & 4620990 & 4614816 \\
\hline 1274 & 540 & 1273 & 4622072 & 4615898 \\
\hline 1275 & 538 & 1274 & 4623150 & 4616976 \\
\hline 1276 & 536 & 1275 & 4624224 & 4618050 \\
\hline 1277 & 534 & 1276 & 4625294 & 4619120 \\
\hline 1278 & 532 & 1277 & 4626360 & 4620186 \\
\hline 1279 & 530 & 1278 & 4627422 & 4621248 \\
\hline 1280 & 528 & 1279 & 4628480 & 4622306 \\
\hline 1281 & 526 & 1280 & 4629534 & 4623360 \\
\hline 1282 & 524 & 1281 & 4630584 & 4624410 \\
\hline 1283 & 522 & 1282 & 4631630 & 4625456 \\
\hline 1284 & 520 & 1283 & 4632672 & 4626498 \\
\hline 1285 & 518 & 1284 & 4633710 & 4627536 \\
\hline 1286 & 516 & 1285 & 4634744 & 4628570 \\
\hline 1287 & 514 & 1286 & 4635774 & 4629600 \\
\hline 1288 & 512 & 1287 & 4636800 & 4630626 \\
\hline 1289 & 510 & 1288 & 4637822 & 4631648 \\
\hline 1290 & 508 & 1289 & 4638840 & 4632666 \\
\hline 1291 & 506 & 1290 & 4639854 & 4633680 \\
\hline 1292 & 504 & 1291 & 4640864 & 4634690 \\
\hline 1293 & 502 & 1292 & 4641870 & 4635696 \\
\hline 1294 & 500 & 1293 & 4642872 & 4636698 \\
\hline 1295 & 498 & 1294 & 4643870 & 4637696 \\
\hline 1296 & 496 & 1295 & 4644864 & 4638690 \\
\hline 1297 & 494 & 1296 & 4645854 & 4639680 \\
\hline 1298 & 492 & 1297 & 4646840 & 4640666 \\
\hline 1299 & 490 & 1298 & 4647822 & 4641648 \\
\hline 1300 & 488 & 1299 & 4648800 & 4642626 \\
\hline 1301 & 486 & 1300 & 4649774 & 4643600 \\
\hline 1302 & 484 & 1301 & 4650744 & 4644570 \\
\hline 1303 & 482 & 1302 & 4651710 & 4645536 \\
\hline 1304 & 480 & 1303 & 4652672 & 4646498 \\
\hline 1305 & 478 & 1304 & 4653630 & 4647456 \\
\hline 1306 & 476 & 1305 & 4654584 & 4648410 \\
\hline 1307 & 474 & 1306 & 4655534 & 4649360 \\
\hline 1308 & 472 & 1307 & 4656480 & 4650306 \\
\hline 1309 & 470 & 1308 & 4657422 & 4651248 \\
\hline 1310 & 468 & 1309 & 4658360 & 4652186 \\
\hline 1311 & 466 & 1310 & 4659294 & 4653120 \\
\hline 1312 & 464 & 1311 & 4660224 & 4654050 \\
\hline 1313 & 462 & 1312 & 4661150 & 4654976 \\
\hline 1314 & 460 & 1313 & 4662072 & 4655898 \\
\hline 1315 & 458 & 1314 & 4662990 & 4656816 \\
\hline 1316 & 456 & 1315 & 4663904 & 4657730 \\
\hline 1317 & 454 & 1316 & 4664814 & 4658640 \\
\hline 1318 & 452 & 1317 & 4665720 & 4659546 \\
\hline 1319 & 450 & 1318 & 4666622 & 4660448 \\
\hline 1320 & 448 & 1319 & 4667520 & 4661346 \\
\hline 1321 & 446 & 1320 & 4668414 & 4662240 \\
\hline & & & & \\
\hline
\end{tabular}

\begin{tabular}{|l|l|l|l|l|}
\hline 1322 & 444 & 1321 & 4669304 & 4663130 \\
\hline 1323 & 442 & 1322 & 4670190 & 4664016 \\
\hline 1324 & 440 & 1323 & 4671072 & 4664898 \\
\hline 1325 & 438 & 1324 & 4671950 & 4665776 \\
\hline 1326 & 436 & 1325 & 4672824 & 4666650 \\
\hline 1327 & 434 & 1326 & 4673694 & 4667520 \\
\hline 1328 & 432 & 1327 & 4674560 & 4668386 \\
\hline 1329 & 430 & 1328 & 4675422 & 4669248 \\
\hline 1330 & 428 & 1329 & 4676280 & 4670106 \\
\hline 1331 & 426 & 1330 & 4677134 & 4670960 \\
\hline 1332 & 424 & 1331 & 4677984 & 4671810 \\
\hline 1333 & 422 & 1332 & 4678830 & 4672656 \\
\hline 1334 & 420 & 1333 & 4679672 & 4673498 \\
\hline 1335 & 418 & 1334 & 4680510 & 4674336 \\
\hline 1336 & 416 & 1335 & 4681344 & 4675170 \\
\hline 1337 & 414 & 1336 & 4682174 & 4676000 \\
\hline 1338 & 412 & 1337 & 4683000 & 4676826 \\
\hline 1339 & 410 & 1338 & 4683822 & 4677648 \\
\hline 1340 & 408 & 1339 & 4684640 & 4678466 \\
\hline 1341 & 406 & 1340 & 4685454 & 4679280 \\
\hline 1342 & 404 & 1341 & 4686264 & 4680090 \\
\hline 1343 & 402 & 1342 & 4687070 & 4680896 \\
\hline 1344 & 400 & 1343 & 4687872 & 4681698 \\
\hline 1345 & 398 & 1344 & 4688670 & 4682496 \\
\hline 1346 & 396 & 1345 & 4689464 & 4683290 \\
\hline 1347 & 394 & 1346 & 4690254 & 4684080 \\
\hline 1348 & 392 & 1347 & 4691040 & 4684866 \\
\hline 1349 & 390 & 1348 & 4691822 & 4685648 \\
\hline 1350 & 388 & 1349 & 4692600 & 4686426 \\
\hline 1351 & 386 & 1350 & 4693374 & 4687200 \\
\hline 1352 & 384 & 1351 & 4694144 & 4687970 \\
\hline 1353 & 382 & 1352 & 4694910 & 4688736 \\
\hline 1354 & 380 & 1353 & 4695672 & 4689498 \\
\hline 1355 & 378 & 1354 & 4696430 & 4690256 \\
\hline 1356 & 376 & 1355 & 4697184 & 4691010 \\
\hline 1357 & 374 & 1356 & 4697934 & 4691760 \\
\hline 1358 & 372 & 1357 & 4698680 & 4692506 \\
\hline 1359 & 370 & 1358 & 4699422 & 4693248 \\
\hline 1360 & 368 & 1359 & 4700160 & 4693986 \\
\hline 1361 & 366 & 1360 & 4700894 & 4694720 \\
\hline 1362 & 364 & 1361 & 4701624 & 4695450 \\
\hline 1363 & 362 & 1362 & 4702350 & 4696176 \\
\hline 1364 & 360 & 1363 & 4703072 & 4696898 \\
\hline 1365 & 358 & 1364 & 4703790 & 4697616 \\
\hline 1366 & 356 & 1365 & 4704504 & 4698330 \\
\hline 1367 & 354 & 1366 & 4705214 & 4699040 \\
\hline 1368 & 352 & 1367 & 4705920 & 4699746 \\
\hline 1369 & 350 & 1368 & 4706622 & 4700448 \\
\hline 1370 & 348 & 1369 & 4707320 & 4701146 \\
\hline 1371 & 346 & 1370 & 4708014 & 4701840 \\
\hline 1372 & 344 & 1371 & 4708704 & 4702530 \\
\hline 1373 & 342 & 1372 & 4709390 & 4703216 \\
\hline 1374 & 340 & 1373 & 4710072 & 4703898 \\
\hline 1375 & 338 & 1374 & 4710750 & 4704576 \\
\hline 1376 & 336 & 1375 & 4711424 & 4705250 \\
\hline 1377 & 334 & 1376 & 4712094 & 4705920 \\
\hline 1378 & 332 & 1377 & 4712760 & 4706586 \\
\hline 1379 & 330 & 1378 & 4713422 & 4707248 \\
\hline & & & & \\
\hline
\end{tabular}




\begin{tabular}{|c|c|c|c|c|c|c|c|c|c|}
\hline 1380 & 328 & 1379 & 4714080 & 4707906 & 1438 & 212 & 1437 & 4745400 & 4739226 \\
\hline 1381 & 326 & 1380 & 4714734 & 4708560 & 1439 & 210 & 1438 & 4745822 & 4739648 \\
\hline 1382 & 324 & 1381 & 4715384 & 4709210 & 1440 & 208 & 1439 & 4746240 & 4740066 \\
\hline 1383 & 322 & 1382 & 4716030 & 4709856 & 1441 & 206 & 1440 & 4746654 & 4740480 \\
\hline 1384 & 320 & 1383 & 4716672 & 4710498 & 1442 & 204 & 1441 & 4747064 & 4740890 \\
\hline 1385 & 318 & 1384 & 4717310 & 4711136 & 1443 & 202 & 1442 & 4747470 & 4741296 \\
\hline 1386 & 316 & 1385 & 4717944 & 4711770 & 1444 & 200 & 1443 & 4747872 & 4741698 \\
\hline 1387 & 314 & 1386 & 4718574 & 4712400 & 1445 & 198 & 1444 & 4748270 & 4742096 \\
\hline 1388 & 312 & 1387 & 4719200 & 4713026 & 1446 & 196 & 1445 & 4748664 & 4742490 \\
\hline 1389 & 310 & 1388 & 4719822 & 4713648 & 1447 & 194 & 1446 & 4749054 & 4742880 \\
\hline 1390 & 308 & 1389 & 4720440 & 4714266 & 1448 & 192 & 1447 & 4749440 & 4743266 \\
\hline 1391 & 306 & 1390 & 4721054 & 4714880 & 1449 & 190 & 1448 & 4749822 & 4743648 \\
\hline 1392 & 304 & 1391 & 4721664 & 4715490 & 1450 & 188 & 1449 & 4750200 & 4744026 \\
\hline 1393 & 302 & 1392 & 4722270 & 4716096 & 1451 & 186 & 1450 & 4750574 & 4744400 \\
\hline 1394 & 300 & 1393 & 4722872 & 4716698 & 1452 & 184 & 1451 & 4750944 & 4744770 \\
\hline 1395 & 298 & 1394 & 4723470 & 4717296 & 1453 & 182 & 1452 & 4751310 & 4745136 \\
\hline 1396 & 296 & 1395 & 4724064 & 4717890 & 1454 & 180 & 1453 & 4751672 & 4745498 \\
\hline 1397 & 294 & 1396 & 4724654 & 4718480 & 1455 & 178 & 1454 & 4752030 & 4745856 \\
\hline 1398 & 292 & 1397 & 4725240 & 4719066 & 1456 & 176 & 1455 & 4752384 & 4746210 \\
\hline 1399 & 290 & 1398 & 4725822 & 4719648 & 1457 & 174 & 1456 & 4752734 & 4746560 \\
\hline 1400 & 288 & 1399 & 4726400 & 4720226 & 1458 & 172 & 1457 & 4753080 & 4746906 \\
\hline 1401 & 286 & 1400 & 4726974 & 4720800 & 1459 & 170 & 1458 & 4753422 & 4747248 \\
\hline 1402 & 284 & 1401 & 4727544 & 4721370 & 1460 & 168 & 1459 & 4753760 & 4747586 \\
\hline 1403 & 282 & 1402 & 4728110 & 4721936 & 1461 & 166 & 1460 & 4754094 & 4747920 \\
\hline 1404 & 280 & 1403 & 4728672 & 4722498 & 1462 & 164 & 1461 & 4754424 & 4748250 \\
\hline 1405 & 278 & 1404 & 4729230 & 4723056 & 1463 & 162 & 1462 & 4754750 & 4748576 \\
\hline 1406 & 276 & 1405 & 4729784 & 4723610 & 1464 & 160 & 1463 & 4755072 & 4748898 \\
\hline 1407 & 274 & 1406 & 4730334 & 4724160 & 1465 & 158 & 1464 & 4755390 & 4749216 \\
\hline 1408 & 272 & 1407 & 4730880 & 4724706 & 1466 & 156 & 1465 & 4755704 & 4749530 \\
\hline 1409 & 270 & 1408 & 4731422 & 4725248 & 1467 & 154 & 1466 & 4756014 & 4749840 \\
\hline 1410 & 268 & 1409 & 4731960 & 4725786 & 1468 & 152 & 1467 & 4756320 & 4750146 \\
\hline 1411 & 266 & 1410 & 4732494 & 4726320 & 1469 & 150 & 1468 & 4756622 & 4750448 \\
\hline 1412 & 264 & 1411 & 4733024 & 4726850 & 1470 & 148 & 1469 & 4756920 & 4750746 \\
\hline 1413 & 262 & 1412 & 4733550 & 4727376 & 1471 & 146 & 1470 & 4757214 & 4751040 \\
\hline 1414 & 260 & 1413 & 4734072 & 4727898 & 1472 & 144 & 1471 & 4757504 & 4751330 \\
\hline 1415 & 258 & 1414 & 4734590 & 4728416 & 1473 & 142 & 1472 & 4757790 & 4751616 \\
\hline 1416 & 256 & 1415 & 4735104 & 4728930 & 1474 & 140 & 1473 & 4758072 & 4751898 \\
\hline 1417 & 254 & 1416 & 4735614 & 4729440 & 1475 & 138 & 1474 & 4758350 & 4752176 \\
\hline 1418 & 252 & 1417 & 4736120 & 4729946 & 1476 & 136 & 1475 & 4758624 & 4752450 \\
\hline 1419 & 250 & 1418 & 4736622 & 4730448 & 1477 & 134 & 1476 & 4758894 & 4752720 \\
\hline 1420 & 248 & 1419 & 4737120 & 4730946 & 1478 & 132 & 1477 & 4759160 & 4752986 \\
\hline 1421 & 246 & 1420 & 4737614 & 4731440 & 1479 & 130 & 1478 & 4759422 & 4753248 \\
\hline 1422 & 244 & 1421 & 4738104 & 4731930 & 1480 & 128 & 1479 & 4759680 & 4753506 \\
\hline 1423 & 242 & 1422 & 4738590 & 4732416 & 1481 & 126 & 1480 & 4759934 & 4753760 \\
\hline 1424 & 240 & 1423 & 4739072 & 4732898 & 1482 & 124 & 1481 & 4760184 & 4754010 \\
\hline 1425 & 238 & 1424 & 4739550 & 4733376 & 1483 & 122 & 1482 & 4760430 & 4754256 \\
\hline 1426 & 236 & 1425 & 4740024 & 4733850 & 1484 & 120 & 1483 & 4760672 & 4754498 \\
\hline 1427 & 234 & 1426 & 4740494 & 4734320 & 1485 & 118 & 1484 & 4760910 & 4754736 \\
\hline 1428 & 232 & 1427 & 4740960 & 4734786 & 1486 & 116 & 1485 & 4761144 & 4754970 \\
\hline 1429 & 230 & 1428 & 4741422 & 4735248 & 1487 & 114 & 1486 & 4761374 & 4755200 \\
\hline 1430 & 228 & 1429 & 4741880 & 4735706 & 1488 & 112 & 1487 & 4761600 & 4755426 \\
\hline 1431 & 226 & 1430 & 4742334 & 4736160 & 1489 & 110 & 1488 & 4761822 & 4755648 \\
\hline 1432 & 224 & 1431 & 4742784 & 4736610 & 1490 & 108 & 1489 & 4762040 & 4755866 \\
\hline 1433 & 222 & 1432 & 4743230 & 4737056 & 1491 & 106 & 1490 & 4762254 & 4756080 \\
\hline 1434 & 220 & 1433 & 4743672 & 4737498 & 1492 & 104 & 1491 & 4762464 & 4756290 \\
\hline 1435 & 218 & 1434 & 4744110 & 4737936 & 1493 & 102 & 1492 & 4762670 & 4756496 \\
\hline 1436 & 216 & 1435 & 4744544 & 4738370 & 1494 & 100 & 1493 & 4762872 & 4756698 \\
\hline 1437 & 214 & 1436 & 4744974 & 4738800 & 1495 & 98 & 1494 & 4763070 & 4756896 \\
\hline
\end{tabular}




\begin{tabular}{|l|l|l|l|l|}
\hline 1496 & 96 & 1495 & 4763264 & 4757090 \\
\hline 1497 & 94 & 1496 & 4763454 & 4757280 \\
\hline 1498 & 92 & 1497 & 4763640 & 4757466 \\
\hline 1499 & 90 & 1498 & 4763822 & 4757648 \\
\hline 1500 & 88 & 1499 & 4764000 & 4757826 \\
\hline 1501 & 86 & 1500 & 4764174 & 4758000 \\
\hline 1502 & 84 & 1501 & 4764344 & 4758170 \\
\hline 1503 & 82 & 1502 & 4764510 & 4758336 \\
\hline 1504 & 80 & 1503 & 4764672 & 4758498 \\
\hline 1505 & 78 & 1504 & 4764830 & 4758656 \\
\hline 1506 & 76 & 1505 & 4764984 & 4758810 \\
\hline 1507 & 74 & 1506 & 4765134 & 4758960 \\
\hline 1508 & 72 & 1507 & 4765280 & 4759106 \\
\hline 1509 & 70 & 1508 & 4765422 & 4759248 \\
\hline 1510 & 68 & 1509 & 4765560 & 4759386 \\
\hline 1511 & 66 & 1510 & 4765694 & 4759520 \\
\hline 1512 & 64 & 1511 & 4765824 & 4759650 \\
\hline 1513 & 62 & 1512 & 4765950 & 4759776 \\
\hline 1514 & 60 & 1513 & 4766072 & 4759898 \\
\hline 1515 & 58 & 1514 & 4766190 & 4760016 \\
\hline 1516 & 56 & 1515 & 4766304 & 4760130 \\
\hline 1517 & 54 & 1516 & 4766414 & 4760240 \\
\hline 1518 & 52 & 1517 & 4766520 & 4760346 \\
\hline 1519 & 50 & 1518 & 4766622 & 4760448 \\
\hline 1520 & 48 & 1519 & 4766720 & 4760546 \\
\hline 1521 & 46 & 1520 & 4766814 & 4760640 \\
\hline 1522 & 44 & 1521 & 4766904 & 4760730 \\
\hline 1523 & 42 & 1522 & 4766990 & 4760816 \\
\hline 1524 & 40 & 1523 & 4767072 & 4760898 \\
\hline 1525 & 38 & 1524 & 4767150 & 4760976 \\
\hline 1526 & 36 & 1525 & 4767224 & 4761050 \\
\hline 1527 & 34 & 1526 & 4767294 & 4761120 \\
\hline 1528 & 32 & 1527 & 4767360 & 4761186 \\
\hline 1529 & 30 & 1528 & 4767422 & 4761248 \\
\hline 1530 & 28 & 1529 & 4767480 & 4761306 \\
\hline 1531 & 26 & 1530 & 4767534 & 4761360 \\
\hline 1532 & 24 & 1531 & 4767584 & 4761410 \\
\hline 1533 & 22 & 1532 & 4767630 & 4761456 \\
\hline 1534 & 20 & 1533 & 4767672 & 4761498 \\
\hline 1535 & 18 & 1534 & 4767710 & 4761536 \\
\hline 1536 & 16 & 1535 & 4767744 & 4761570 \\
\hline 1537 & 14 & 1536 & 4767774 & 4761600 \\
\hline 1538 & 12 & 1537 & 4767800 & 4761626 \\
\hline 1539 & 10 & 1538 & 4767822 & 4761648 \\
\hline 1540 & 8 & 1539 & 4767840 & 4761666 \\
\hline 1541 & 6 & 1540 & 4767854 & 4761680 \\
\hline 1542 & 4 & 1541 & 4767864 & 4761690 \\
\hline 1543 & 2 & 1542 & 4767870 & 4761696 \\
\hline & & & & \\
\hline
\end{tabular}

Thus, it is seen that there are 514 pairs of Pythagorean triangles such that for each pair the difference in the perimeters is 4- digit Dhuruva number 6174.

Out of these 6174 pairs of Pythagorean triangles 1-pair is non-primitive and in each of the remaining pairs, one of the triangles is primitive and the other is non-primitive triangle. A similar observation, regarding 5- digit and 6- digit dhuruva numbers are exhibited in the table 2 below.

\begin{tabular}{|l|l|l|l|}
\hline $\begin{array}{l}\text { Dhurua } \\
\text { number }\end{array}$ & $\begin{array}{l}\text { pairs of } \\
\text { Pythagorean } \\
\text { triangles }\end{array}$ & $\begin{array}{l}\text { pairs of } \\
\text { non- } \\
\text { primitve } \\
\text { Pythagoren } \\
\text { triangles }\end{array}$ & $\begin{array}{l}\text { pairs of primitve } \\
\text { and non- } \\
\text { primitve } \\
\text { Pythagorean } \\
\text { triangles }\end{array}$ \\
\hline 61974 & 5164 & 62 & 5102 \\
\hline 82962 & 6913 & 1391 & 5522 \\
\hline 63954 & 5329 & 439 & 4890 \\
\hline 631764 & 52647 & - & 52647 \\
\hline
\end{tabular}

\section{CONCLUSION}

In this paper, we have presented pairs of Pythagorean triangles such that, in each pair, the difference between their perimeters is represented by Dhuruva numbers of orders 4,5 and 6. It is observed that there are only finitely many Pythagorean triangles satisfying the property under consideration. Also we have given the total number of pairs of Primitive and non-primitive Pythagorean triangles.

To conclude, One may search for the connections between the pairs of Pythagorean triangles, higher order Dhuruva numbers, Special polygonal and pyramidal numbers. Also one may attempt to determine pairs of Pythagorean triangles, where, in each pair, the difference between their areas is represented by Dhuruva numbers, figurate numbers and other special number patterns, namely, Star numbers, Jacobstal numbers, Kynea numbers and so on.

\section{ACKNOWLEDGEMENTS}

The financial support from the UGC, New Delhi (F-MRP$5122 / 14$ (SERO/UGC) dated march 2014) for a part of this work is gratefully acknowledged.

\section{REFERENCES}

[1] W.Sierpinski, Pythagorean triangles, Dover publications, INC, New York, 2003.

[2] M.A.Gopalan and G.Janaki,'Pythagorean triangle with area/perimeter as a special polygonal number", Bulletin of Pure and Applied Science, Vol.27E (No.2), 393-402,2008.

[3] M.A.Gopalan and A.Vijayasankar, "Observations on a Pythagorean problem", ActaCienciaIndica, Vol.XXXVI M, No 4, 517-520, 2010.

[4] M.A.Gopalan and S.Leelavathi, "Pythagorean triangle with area/perimeter as a square integer", International Journal of Mathemtics, Computer sciences and Information Technology, Vol.1, No.2, 199-204, 2008.

M.A.Gopalan and A.Gnanam,"Pairs of Pythagorean triangles with equal perimeters", Impact J.Sci.Tech., Vol 1(2), 67-70, 2007.

[6] M.A.Gopalan and S.Leelavathi, "Pythagorean triangle with 2 area/perimeter as a cubic integer", Bulletin of Pure and Applied Science, Vol.26E(No.2), 197-200,2007.

[7] M.A.Gopalan and A.Gnanam,"A special Pythagorean problem", ActaCienciaIndica, Vol.XXXIII M, No 4, 1435-1439,2007. 
[8] M.A.Gopalan, A.Gnanam and G.Janaki,”A Remarkable Pythagorean problem",ActaCienciaIndica, Vol.XXXIII M, No 4, 1429-1434,2007.

[9] M.A.Gopalan, and S.Devibala,"On a Pythagorean problem",ActaCienciaIndica, Vol.XXXII M, No 4, 1451-1452,2006.

[10] M.A.Gopalan and B.Sivakami,"Special Pythagorean triangles generated through the integral solutions of the equation $y^{2}=\left(k^{2}+2 k\right) x^{2}+1$ ", Diophantus J.Math., Vol 2(1), 25-30, 2013.

[11] M.A.Gopalan and A.Gnanam,"Pythagorean triangles and Polygonal numbers", International Journal of Mathematical Sciences, Vol 9, No. 1-2,211215,2010.

[12] K.Meena, S.Vidhyalakshmi, B.Geetha, A.Vijayasankar and M.A.Gopalan,'Relations between special polygonal numbers generated through the solutions of Pythagorean equation", IJISM, Vol 5(2), 15-18, 2008.

[13] M.A.Gopalan and G.Janaki,'Pythagorean triangle with perimeter as Pentagonal number", AntarticaJ.Math., Vol 5(2), 15-18,2008.

[14] M.A.Gopalan and G.Sangeetha,'Pythagorean triangle with perimeter as triangular number", GJAMMS, Vol. 3, No 1-2, 93-97,2010.

[15] M.A.Gopalan, Manjusomanath and K.Geetha,'Pythagorean triangle with area/perimeter as a special polygonal number",IOSR-JM, Vol.7(3),52-62,2013.

[16] M.A.Gopalan and V.Geetha,'Pythagorean triangle with area/perimeter as a special polygonal number",IRJES,Vol.2(7),28-34,2013.

[17] M.A.Gopalan and B.Sivakami,'Pythagorean triangle with hypotenuse minus 2(area/perimeter) as a square integer",ArchimedesJ.Math., Vol 2(2),153166,2012 .

[18] J.N.Kapur, Dhuruva numbers, Fascinating world of Mathematics and Mathematical sciences, Trust society, Vol 17,1997.

[19] Bert Miller, Nasty numbers, The mathematics teacher, No.9, Vol 73,649,1980.

[20] Charles Bown.K, Nasties are primitives, The mathematics teacher, No.9, Vol 74,502-504,1981.

[21] P.S.N.Sastry, Jarasandha numbers, The mathematics teacher, No.9,Vol 37,issues 3 and 4,2001.

[22] M.A.GopalanV.Sangeetha and Manjusomanath," Pythagorean triangle and Polygonal number", CayleyJ.Math., Vol 2(2),151-156,2013.

[23] M.A.Gopalan and G.Janaki,"pythagorean triangle with nasty number as a leg",Journal of applied Mathematical Analysis and Applications, Vol 4,No 1-2,13-17,2008.

[24] M.A.Gopalan and S.Devibala,"Pythagorean triangle with triangular number as a leg", ImpactJ.Sci.Tech., Vol 2(4), 195-199,2008.

[25] Dr.MitaDarbari, A connection between HardyRamanujan number and special Pythagorean triangle," Bulletin of society for Mathematical services and standards, Vol 3, No.2, 71-73, 2014.
[26] M.A.Gopalan , S.Vidhyalaksmi, E.Premalatha and R.Presenna, "Special Pythagorean triangles and 5digit dhuruva numbers".IRJMEIT, Vol 1(4),2933,Aug 2014. 